\title{
Sistema de Perfeccionamiento Orientado a Competencias para Docentes de la Educación Superior
}

\author{
Fredi E. Palominos ${ }^{(1) \star}$, Miguel A. Mendez ${ }^{(2)}$ y Rosa A. Barrera(1) \\ (1) Departamento de Matemática y Ciencia de la Computación, Universidad de Santiago de Chile, Avenida \\ Las Sophoras № 175, Comuna de Estación Central, Santiago, Chile. \\ (2) Coordinador de Gestión Curricular Dirección de Posgrado, Universidad de Santiago de Chile, Avenida \\ Las Sophoras ํㅜ 175, Comuna de Estación Central, Santiago, Chile. \\ (e-mail: fredi.palominos@usach.cl,miguel.mendez.ferrada@gmail.com,rosa.barrera@usach.cl)
}

* Autor a quien debe ser dirigida la correspondencia.

Recibido Sep. 11, 2013; Aceptado Oct. 4, 2013; Versión final recibida Ene. 15, 2014

\begin{abstract}
Resumen
El trabajo expone un sistema de perfeccionamiento orientado a competencias para docentes de la educación superior, destinado a apoyar los procesos de modernización de la docencia en pre y postgrado. A partir de diferentes experiencias, se identifican factores que frecuentemente han afectado negativamente o impedido el éxito del perfeccionamiento y la innovación. Los diversos elementos del sistema tienen por finalidad neutralizar o disminuir estos factores, valiéndose para ello de un proceso de diagnóstico adecuadamente contextualizado, la identificación de metas significativas y la planificación del proceso, que en armonía con la política institucional, permita generar las condiciones adecuadas para la innovación y el cambio. Los resultados evidencian que el sistema permite identificar adecuadamente las necesidades de perfeccionamiento, considerando los factores culturales de la institución.
\end{abstract}

Palabras clave: perfeccionamiento docente, competencias, modernización curricular, educación superior, educación

\section{Oriented Skill System of Continuing Education for Teachers of Higher Education}

\begin{abstract}
The paper presents an oriented skill system of continuing education for teachers of higher education intended to support the modernization of teaching in undergraduate and graduate levels. Considering several previous experiences, factors that often adversely affected or impeded the success of the development and innovation are identified. The various elements of the system are designed to neutralize or reduce these factors, relying on a diagnostic process properly contextualized, identifying meaningful goals and process planning which in agreement with institutional policy allow generating the appropriate conditions for innovation and change. The results show that the system can properly identify development needs, considering the cultural factors of the institution.
\end{abstract}

Keywords: teaching development, skills, curricular modernization, higher education, education 


\section{INTRODUCCIÓN}

La educación basada en competencias tiene varias décadas de existencia (Corvalán, 2008), sin embargo, su introducción en Chile, es relativamente reciente. Diversas universidades nacionales han utilizado este enfoque como una vía para enfrentar los procesos de renovación curricular (Pey, 2011), no obstante, los resultados no son concluyentes, debido a que comprenden experiencias que van entre aquellas que más bien parecen "procesos cosméticos" para asemejarse a las universidades más vanguardistas, hasta trabajos serios y profundos, que han implicado transformaciones importantes en el currículo de muchas carreras. Por otra parte, a la expectativa de la educación basada en competencias, se suma la cada vez mayor entronización de las Tecnologías de Información (TI) (OECD, 2012), que en la educación superior ha generado un contexto potencialmente más productivo y eficiente, que para muchos es la promesa de una nueva educación, más personalizada, más efectiva, más profunda, más centrada en el estudiante y con roles más explícitos y bien definidos (Montero, 2008; Trujillo, 2006).

Así, como en todos los ámbitos de la innovación, la experticia, la racionalidad y la correcta planificación son factores determinantes. La debilidad o el descuido de uno o más de estos factores han derivado en que diversas iniciativas innovadores, motivadas por la promesa de la educación basada en competencias, no hayan tenido los resultados esperados (Cavallo 2004), llevando a la desaceleración o el congelamiento de proyectos de modernización, el desencanto de los participantes y de las comunidades académicas (Jiménez, 2010; Camperos, 2008) en el modelo basado en competencias.

Las experiencias negativas, se pueden atribuir principalmente a que:

1) El mito de que la simple introducción del concepto de competencia resuelve "mágicamente" los problemas metodológicos, las debilidades del currículo y/o la de planificación de la docencia (Tardif, 2003). En efecto, dado que este falso paradigma es simplemente una reducción simplista del problema, quienes se confiaron en estas hipótesis incorrectas, hoy sostienen que la educación basada en competencias es sólo un mito, una moda o simplemente que no es la respuesta para acceder a nuevos y mejores itinerarios de formación académica.

2) Con frecuencia, los procesos de renovación curricular se realizan sin los asesoramientos técnicos adecuados o bien soslayando o minimizando procesos vitales como la construcción, actualización o validación del perfil profesional o el perfeccionamiento del cuerpo académico. De esta forma, la renovación curricular se reduce a la introducción de información en formularios (generalmente orientados a asignaturas), donde los participantes (generalmente profesores) aportan desde la perspectiva disciplinaria visiones parciales y sesgadas, de modo que los viejos y tradicionales objetivos de asignaturas, se maquillan como competencias profesionales o cognitivas (Angulo, 2011; Guzmán, 2011).

3) En ocasiones, la renovación curricular en el enfoque orientado a competencias, no cuentan con el apoyo institucional (Moyano, 2012) y sólo está sustentada en el compromiso y el entusiasmo de grupos de académicos.

4) Con frecuencia se confunde el concepto y el desarrollo de competencias, con el desarrollo de habilidades (Climént, 2010).

5) Se introduce parcialmente el enfoque orientado a competencias y no se consideran cambios en los sistemas de evaluación. Formar por competencias requiere revisar profundamente o replantearse los tradicionales enfoques evaluativos, avanzando hacia nuevos enfoques que incorporen más tipos de instrumentos evaluativos, más precisos y mejor focalizados, libres del sesgo del contexto o de las percepciones o pre concepciones personales de profesor (Scallon, 2004; González, 2005).

6) Existe un inmovilismo latente en diversas comunidades académicas, donde el profesor es reacio a introducir cambios que afecten su rol, sus creencias y prácticas profesionales (Del Valle, 2012).

Si a lo anterior se suman altas expectativas y falsas creencias, como por ejemplo las que han surgido en torno al uso de TI (UNESCO 2005), la confusión puede ser aún mayor (Edwards, 2003). En efecto, con frecuencia se pensó que la sola inclusión de las TI aportaría grandes soluciones, como por ejemplo:

a) Alumnos más autónomos y mejor preparados para aprender a aprender. No obstante, no se consideró que este cambio conductual requiere una profunda revisión de la enseñanza tradicional, una tutoría acorde con los conocimientos a trasmitir, profesores y/o tutores formados y preparados para estos nuevos desafíos, 
condiciones tecnológicas adecuadas, recursos tecnológicos bien implementados y un sólido, evidente y consecuente respaldo institucional (Palominos, 2008).

b) Menor esfuerzo para el profesor, sustentado en la reducción de las sesiones de clases presenciales y en la "automatización" de la aplicación y revisión de evaluaciones. Apreciación errónea desde todo ángulo, debido a que tanto la educación basada en competencias como la introducción de TI, debido al cambio de roles, requieren de un mayor tiempo de dedicación del profesor, (Palominos, 2008), para dedicarse a tareas cotidianas como: dar respuesta a consultas en línea, monitorear foros, preparar o seleccionar material multimedial.

Si además sumamos los factores culturales propios de cada nación, estado, colectivo o institución, que siempre deben considerarse en procesos de renovación curricular y en la introducción de innovaciones, implica que las estrategias y soluciones requeridas por una institución y contexto, son especiales y específicas, por lo que probablemente son también únicas. No obstante este escenario, la educación basada en competencias y la natural, necesaria, obligatoria e irrenunciable articulación con las TI, seguirán constituyendo un desafío ineludible (Cavallo, 2004; Montero 2008). No es posible ignorar que actualmente diversos elementos externos, la globalización, los mercados de trabajo y los procesos de formación profesional requieren la certificación de estándares y que la educación basada en competencias, es una respuesta estas necesidades (Ramírez, 2008).

\section{Las competencias y su relación con el perfeccionamiento académico}

Una competencia, es un "saber actuar complejo fundado sobre la movilización y la combinación eficaces de un conjunto de recursos (internos y externos) en una familia de situaciones" (Tardif, 2003). De esta forma, la adquisición de una competencia se debe evidenciar mediante la capacidad de una persona para orquestar un conjunto de atributos personales, tales como: conocimientos, habilidades, destrezas, intereses, actitudes, valores y otras disposiciones, en el logro o resolución exitosa de una tarea o problema existente en una situación del mundo real. En el ámbito de la formación profesional y en particular en la educación superior, la definición, formación y evaluación de competencias profesionales de egreso, requiere de una metodología que recoja la multidimensionalidad del desarrollo de las personas en el aprendizaje de una profesión.

\section{ROL DEL PERFECCIONAMIENTO}

En el sistema educativo formal, los diferentes actores interactúan en diversas instancias, cumpliendo roles que en la enseñanza tradicional han permanecido relativamente estables por décadas (Pedraja, 2012; Herrera, 20013; Muñoz, 2012). En el caso específico de la educación superior, los más proclives a los cambios son los estudiantes, situación que se justifica probablemente por la menor familiaridad que tienen con este tipo de sistema educativo y por la fuerte inhibición que pueda producir la relación profesor alumno.

La innovación o la renovación del currículo en el enfoque orientado a competencias, requieren un tratamiento sistémico. Los cambios que implica a nivel del proceso de enseñanza aprendizaje van mucho más allá del aula y alcanzan a todo el sistema educativo. A nivel administrativo, los cambios se traducen en ajustes reglamentarios o cambios de procedimientos, pero a medida que se penetra en el nivel académicodocente, se requiere una redefinición del mapa conceptual de los profesores, a fin de que no solamente acepten el nuevo paradigma, sino a que además lo hagan parte de sus creencias. Este cambio de paradigma es un desafío significativo, debido a que no solo afecta al deber ser del profesor, sino que incide en la planificación y la gestión de la docencia, en los reglamentos y los itinerarios de formación, en la relación de la institución con el medio, en las autoridades universitarias y en los estudiantes. En consecuencia, el perfeccionamiento del sistema que radica fundamentalmente en el perfeccionamiento de los actores, hace que este último sea de vital importancia para el éxito de un proceso de innovación. La presente propuesta se hace cargo de estos factores claves, a través de un proceso de perfeccionamiento focalizado, acorde al perfil de cada actor del sistema.

\section{SISTEMA DE PERFECCIONAMIENTO}

El objetivo de este trabajo es proponer las bases de un sistema de perfeccionamiento para docentes de la educación superior, en el ámbito del enfoque orientado a competencias, conforme al enfoque de (Tardif, 2003), que haciéndose cargo de los roles de los diferentes actores, en especial los docentes, brinde un marco de referencia que permita implementar una metodología de perfeccionamiento capaz de apoyar los procesos de modernización de la docencia o renovación curricular de programas de estudio de pre y postgrado, a nivel de una carrera, departamento o escuela, facultad o toda la institución. 
Como se expresa en secciones anteriores, el éxito de cualquier proceso de modernización o renovación curricular en base al enfoque orientado a competencias, depende en una buena medida del perfeccionamiento del estamento académico y de la capacitación del estamento administrativo y profesional. El sistema propuesto en este trabajo se traduce en una metodología para enfrentar el perfeccionamiento, que responde a un enfoque sistémico. El sistema se fundamenta en que el perfeccionamiento debe permitir a los docentes iniciarse en una práctica profesional reflexiva, situada en el contexto de un sistema de enseñanza reflexiva; "sólo si se desarrolla en el profesor la capacidad de reflexión crítica y de autodirección, éste podrá repensar su teoría implícita sobre la enseñanza, sus actitudes hacia la docencia y hacia sus estudiantes, lo que facilitará una reconstrucción de su visión y eventual actuación en el aula" (Díaz, 2002), sin olvidar que el conocimiento profesional de los profesores se articula en los planos conceptual, práctico y reflexivo.

En un contexto como el descrito en este trabajo, el perfeccionamiento y la innovación deben ser liderados y coordinados por un grupo de académicos, que deben ser respaldados por las autoridades universitarias a distintos niveles. Denominaremos diseñadores del proceso de formación, al grupo de especialistas que liderarán, analizarán, diseñarán e implementarán un nuevo plan de estudios o una nueva versión del mismo. Este grupo es crucial dentro del proceso de modernización debido a que debe construir o participar en la construcción del perfil de egreso, definir los itinerarios de formación para los estudiantes (hipótesis evolutiva), definir los aportes de cada una de las asignaturas al proceso de formación, los posibles mecanismos de aseguramiento de la calidad y el monitoreo del proceso de implantación de las innovaciones.

\section{Objetivos del Sistema}

El sistema propuesto en este trabajo, contribuye a la superación de los problemas previamente descritos, debido a que se fundamenta en el diagnóstico del contexto y la inclusión de diferentes componentes sistémicas claves para el perfeccionamiento, la renovación curricular y la introducción de innovaciones en la docencia.

En el ámbito de la contextualización del perfeccionamiento (Pentland, 2004; Trujillo, 2006; Montero, 2008A), el sistema: (1) Promueve una adecuada contextualización del concepto de competencia, diferenciándolo de aspectos metodológicos propios de la docencia, (2) Considera los factores culturales propios de la institución educativa, incorporando un diagnóstico del contexto en el cual se desarrollará el proceso de renovación curricular, y (3) Contempla una adecuada contextualización de las TI, conforme a las necesidades del perfeccionamiento del proceso de renovación curricular, sin exacerbar su rol y protagonismo, de modo que permita a los participantes utilizarla con la mayor soltura, confianza y naturalidad posible.

En el ámbito del diagnóstico y la planificación del perfeccionamiento, el sistema: (1) Concibe el perfeccionamiento como un proceso planificado, focalizado y realista, que considera las características de los actores y las áreas a intervenir, conforme al perfil profesional deseado, (2) Considera una adecuada planificación de los tiempos y de los recursos, acorde con las expectativas y el contexto de la institución, (3) Considera una adecuada socialización de las metas y el plan de trabajo en la comunidad académica, (4) Enfatiza de forma explícita la necesidad de asegurar la disponibilidad de recursos, en los tiempos oportunos, conforme a los requerimientos del proceso de perfeccionamiento, y (5) Considera la disponibilidad de tiempo de dedicación de los participantes.

En el ámbito del perfeccionamiento, el sistema: (1) Propone un perfeccionamiento docente focalizado en el rol que el profesor debe cumplir en la enseñanza y su disposición frente al proceso de renovación curricular, de modo que el fruto del perfeccionamiento sean los conocimientos, habilidades y actitudes que mejoren el desempeño del docente, que le permitan una actuar competente en el proceso de cambio o modernización, y (2) Enfatiza la necesidad de diferenciar claramente los conceptos de competencia, habilidad, actitud, indicadores y criterios evaluativos, incorporando la evaluación de competencias como parte fundamental del proceso de perfeccionamiento.

\section{Contribuciones del Sistema de Perfeccionamiento}

El sistema de perfeccionamiento permite facilitar los procesos de diseño y rediseño curricular, así como su posterior implementación, debido a que posibilita las siguientes acciones: a) Eliminar, minimizar o neutralizar los factores culturales que se oponen a la modernización y a la innovación, factores que habitualmente se originan en el desconocimiento y la incertidumbre que estos procesos imprimen en los actores del sistema educativo; b) Generar condiciones para lograr una mejor articulación de los planes de estudio, con programas de asignaturas mejor focalizadas, más acordes con el perfil de egreso y las expectativas del 
mercado laboral, tanto en el ámbito de la formación profesional como en el de la formación científica; c) Que los docentes y quienes gestionan la docencia, logren: (i) Una mejor comprensión del concepto de competencia y la educación basada en competencias, así como las diferencias que existen entre los tradicionales objetivos de aprendizaje y las competencias profesionales, (ii) Un nivel de perfeccionamiento que le permita contribuir de forma efectiva en la implementación de los procesos de renovación curricular, comprendiendo la contribución de su asignatura al perfil de egreso, y (iii) Una mejor actitud y familiaridad hacia las TI; d) Contempla que los responsables y ejecutores del proceso de renovación curricular, se perfeccionen a fin de conseguir un nivel de perfeccionamiento que les permita un desempeño competente en el diseño o rediseño curricular del plan de estudios, conforme al perfil de egreso de la carrera.

\section{Descripción del Sistema}

El sistema consta de diversas componentes que se articulan en torno a tres aspectos fundamentales denominados: (a) Diagnóstico, (b) Planificación y (c) Perfeccionamiento (ver figura 1). Desde el punto de vista metodológico, estos tres aspectos son la esencia del sistema, por cuanto orientan y delinean la ejecución del proceso de perfeccionamiento.

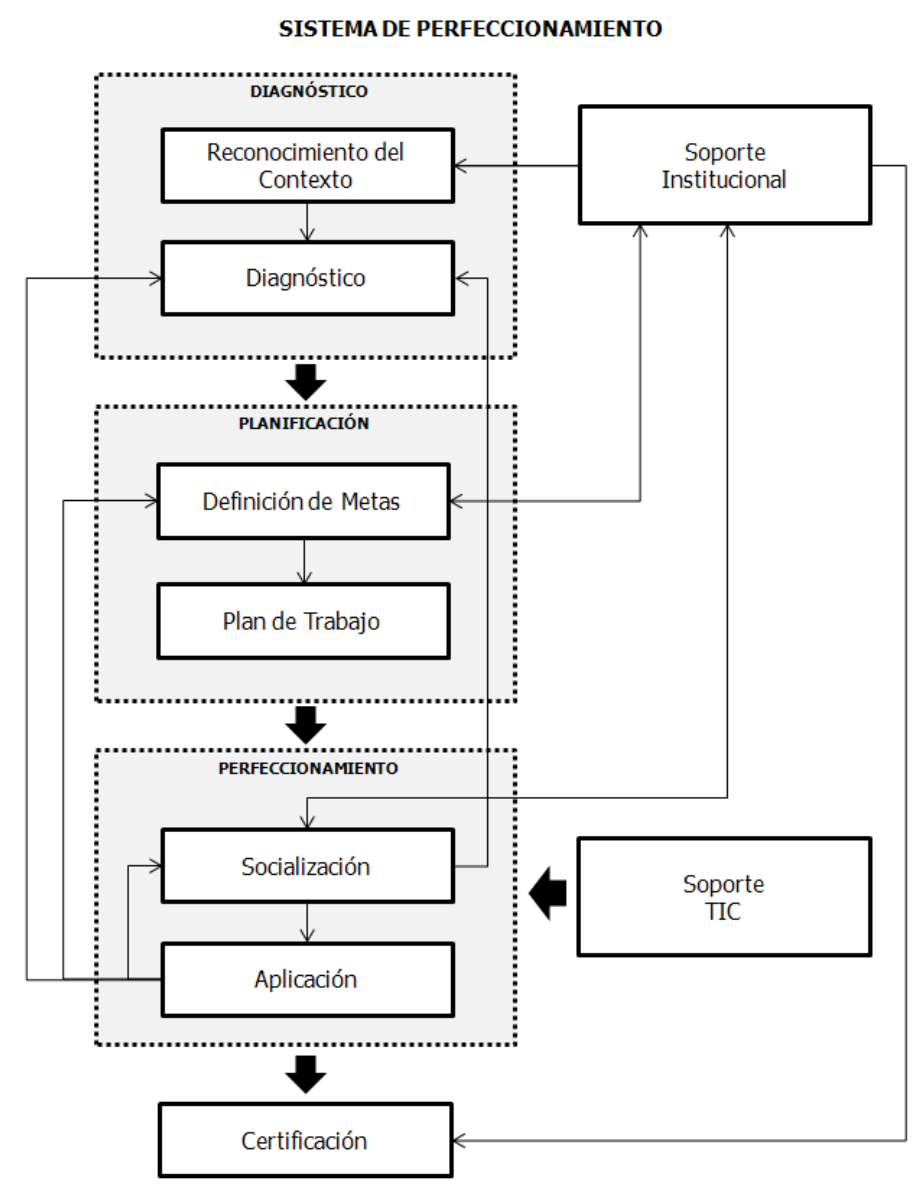

Fig. 1: Sistema de Perfeccionamiento Orientado a Competencias (SPOC).

Dado su carácter integral, el sistema también contempla la participación de personas con influencia o poder de decisión (autoridades) en la institución, grupo al que se denomina Soporte Institucional. Ellos cumplen el rol de gestionar o proveer todo el apoyo logístico y recursos necesarios para la realización del proceso de perfeccionamiento, así como dar el respaldo explícito de la institución ante la comunidad académica y administrativa. Otra componente del sistema, denominada Soporte TIC, involucra a los expertos en TIC y a los especialistas en informática. Comprende además el equipamiento informático y los recursos multimediales requeridos por los diferentes participantes en el proceso de perfeccionamiento. La misión de los expertos en TIC, es integrar adecuadamente la tecnología, teniendo en cuenta las características de las herramientas disponibles, así como las habilidades y pre concepciones de los participantes (Lewis, 2005; Edwards, 2003). Finalmente, el sistema contempla que el proceso de perfeccionamiento concluya con el reconocimiento institucional del esfuerzo y compromiso asumido por el cuerpo académico y administrativo, etapa que se denomina Certificación. 


\section{Usuarios del Sistema de Perfeccionamiento}

El sistema de perfeccionamiento tiene diversos tipos de usuarios, siendo los principales los siguientes: (1) Los docentes, quienes son beneficiarios directos del sistema al ser receptores del perfeccionamiento, (2) Los estudiantes, quienes son los beneficiarios indirectos del sistema a través de la mejora en las prácticas docentes, (3) Los gestores de los programas de estudios, quienes son beneficiarios directos del sistema y se perfeccionan para liderar el proceso de renovación curricular y en particular el perfeccionamiento de los docentes, (4) Los directivos, quienes son beneficiarios indirectos del sistema y deben respaldar el perfeccionamiento, así como propiciar los cambios reglamentarios y de procedimientos que sustenten los cambios en la gestión curricular y el ejercicio docente, y (5) El estamento administrativo, quienes son beneficiarios indirectos del sistema y deben, conforme a los cambios reglamentarios y de procedimientos, apoyar la gestión curricular y la innovación en el ejercicio docente.

\section{DESCRIPCIÓN DE LAS COMPONENTES DEL SISTEMA}

\section{Subsistema de Diagnóstico}

El subsistema diagnóstico tiene por finalidad tomar conocimiento del contexto en el cual se realizará el proceso de perfeccionamiento o innovación, considerando elementos como: (a) aspiraciones institucionales y de quienes lideran el proceso, (b) proyecto de renovación curricular (si existiere), (c) creencias de la comunidad académica (docentes, administrativos y estudiantes), (d) experiencia en actividades de innovación, (e) disponibilidad de recursos, (f) apoyo institucional, etc.

El subsistema de diagnóstico debe entregar información precisa respecto del contexto, identificando aquellos elementos que quienes lideran el proceso consideran relevantes, las eventuales amenazas, los recursos requeridos y las fortalezas de la comunidad académica. Esta etapa de diagnóstico permitirá validar el proyecto de innovación curricular y los objetivos del perfeccionamiento académico, permitiendo introducir ajustes o mejoras en la planificación. Esta etapa debería proporcionar la información necesaria para descartar aquellos elementos que no aportan o pueden constituir una amenaza para el perfeccionamiento. El subsistema de diagnóstico consta de la realización de dos etapas, que se articulan de forma secuencial. Como resultado, aporta las metas del proceso de perfeccionamiento o bien la validación, modificación o redefinición de las mismas, si hubieren sido definidas con anterioridad. Dichas componentes son las siguientes:

\section{Reconocimiento del Contexto}

El reconocimiento y entendimiento del contexto en el cual se desarrollará el perfeccionamiento, es uno de los pilares del sistema. Este proceso tiene por objetivo reunir información de contexto e identificar las características esenciales que definen las creencias de la comunidad académica y las metas declaradas o implícitas en el proyecto de innovación o perfeccionamiento. Durante esta etapa, se deberán identificar, al menos, los siguientes elementos: 1) Aspiraciones de la comunidad académica respecto de la modernización curricular o el perfeccionamiento académico; 2) Visión del equipo encargado de la modernización curricular o el perfeccionamiento docente; 3) Opiniones de la comunidad académica (docentes, administrativos y estudiantes) respecto del proyecto de renovación curricular o el perfeccionamiento; 4) Elementos culturales propios de la comunidad académica o de la institución, que influyen o pueden influir en el proceso de perfeccionamiento; 5) Visión y grado de compromiso de las autoridades universitarias respecto del proceso.

Está etapa del proceso, es particularmente dependiente de una asesoría experta y un apoyo institucional explícito, a fin de que la comunidad académica no dude respecto de la seriedad e importancia de la participación en la misma.

\section{Diagnóstico}

En base a los elementos de contexto identificados en el proceso de reconocimiento descrito previamente, se debe elaborar un diagnóstico de la situación actual de la comunidad académica y del proyecto de renovación curricular o perfeccionamiento. Para que el diagnostico sea pertinente al proceso, debe incluir: (1) Fortalezas y debilidades de la comunidad académica y la institución, haciendo especial énfasis en aquellos logros o aspectos positivos que deben permanecer o fortalecerse, y aquellos que son debilidades 0 potenciales amenazas para la comunidad académica, el plan de estudios o la institución, (2) La relevancia y pertinencia del proyecto de modernización o perfeccionamiento (si existiere), respecto de las necesidades de perfeccionamiento y aspiraciones de la comunidad académica detectadas en el diagnóstico, (3) La identificación, definición y selección de las reales necesidades de perfeccionamiento, (4) Las condiciones 
técnicas necesarias para la realización de proyecto de modernización curricular, (5) Los aportes y recursos requeridos para potenciar el proyecto de renovación curricular o perfeccionamiento de la unidad académica, en el contexto identificado, y (6) Una adecuada definición de los contenidos de perfeccionamiento y la identificación de los diferentes actores que serán sujetos del mismo.

\section{SUBSISTEMA DE PLANIFICACIÓN}

Tiene por finalidad establecer las metas del proceso de perfeccionamiento y un plan de trabajo para el mismo. En el caso que la comunidad académica cuente con un proyecto de innovación o perfeccionamiento previo, permitirá validar la pertinencia del proyecto y eventualmente adecuar las metas previamente establecidas. Los procesos asociados al subsistema de planificación son:

\section{Definición de Metas}

En base al diagnóstico elaborado en la etapa anterior, las expectativas formuladas por el equipo que lidera el proceso de innovación o perfeccionamiento y las metas declaradas por la institución, se formularán un conjunto de metas viables para dicho contexto. Es imprescindible que dichas metas estén en conocimiento y cuenten con el apoyo de la componente de soporte institucional. Además, deben socializarse adecuadamente, a fin de que sean correctamente percibidas y conocidas por la comunidad académica. El resultado de este proceso entrega: (1) Las mestas, objetivos generales y objetivos específicos del proceso de innovación o perfeccionamiento, así como eventuales alternativas y mejoras al proyecto de modernización curricular de la unidad académica, si existiere, (2) La definición de los roles que deberán cumplir los miembros de la comunidad académica, y (3) La definición de las instancias de apoyo que deberá brindar el equipo de soporte institucional.

\section{Plan de Trabajo}

En la elaboración del plan de trabajo, como en toda herramienta de planificación y gestión, se debe formular en función de las metas previamente establecidas en la etapa previa. Debido a que las metas representan y reflejan las aspiraciones de la comunidad académica, así como de la institución, ellas deben ser operativas en términos de objetivos, actividades, indicadores de logros, resultados y productos. El plan de trabajo es la guía para llevar a cabo el trabajo durante un periodo de tiempo definido, proporcionando los elementos de verificación, identificando los recursos requeridos y los momentos en que son necesarios y se debe asegurar su disponibilidad. Como tal, debe ser conocido y aprobado institucionalmente, de modo que cuente con los recursos requeridos y el respaldo explícito ante la comunidad académica.

\section{SUBSISTEMA DE PERFECCIONAMIENTO}

Tiene por finalidad la correcta articulación y aplicación del perfeccionamiento, por tanto contempla diversos aspectos, que incluyen la socialización y la ejecución del proceso en sí mismo. El proceso de perfeccionamiento es un conjunto de acciones que tienen por finalidad lograr que cada actor del sistema educativo, alcance el nivel de competencia requerido para cumplir adecuadamente con su rol en el nuevo contexto de la innovación. Por ende, el proceso de perfeccionamiento es mucho más que la simple suma de un conjunto de cursos. El sistema presentado en este trabajo, se hace cargo de una necesidad que frecuentemente no se considera, como es la socialización del proceso, aspecto clave para el éxito de la innovación curricular. En el sistema, la socialización y el perfeccionamiento se articulan de forma secuencial.

\section{Socialización del Proceso}

La participación informada de los diferentes actores, es muy relevante para el éxito de la innovación y el perfeccionamiento. Esta condición puede lograrse a través de una adecuada planificación del proceso de socialización, oportunidad en el que no sólo se transmiten las aspiraciones de quienes impulsan el proceso de renovación curricular o perfeccionamiento, sino que se atiende las demandas e inquietudes de todos los participantes, a fin de lograr la confianza de los diferentes estamentos. Entre los grupos de actores, donde preferentemente se deben realizar actividades de socialización, destacan los siguientes:

\section{Socialización en el ámbito Institucional y Directivo}

Debido a que las autoridades son quienes deben dar el respaldo y en ocasiones impulsar el proceso de cambio, cumplen un rol fundamental en el proceso de perfeccionamiento necesario para la modernización curricular de un programa de estudio y deben permanecer visibles e involucradas en el proceso de 
modernización. Por esta razón, deben tener un adecuado conocimiento del plan de perfeccionamiento y/o el proyecto de innovación.

La socialización de proceso a nivel de las autoridades, debe al menos considerar los siguientes aspectos: (1) Se debe asegurar que los directivos tengan claridad sobre la relevancia que tienen para el proceso, de modo que los diferentes actores perciban en la autoridad respaldo, interés y presencia., (2) Los directivos deben conocer los objetivos del proceso de modernización curricular del programa, (3) Los directivos deben conocer el plan de trabajo, en especial aquellos hitos que están asociados a las acciones que ellos deben realizar para asegurar la disponibilidad de recursos, y (4) Los directivos deben conocer los cambios reglamentarios requeridos por el proceso de modernización, los cuales deberán apoyar o tramitar en los momentos que corresponda.

\section{Socialización en el ámbito Estudiantil}

Los estudiantes de una carrera o plan de estudios, generalmente tienen un conocimiento limitado de los fundamentos que originaron el currículo del plan de estudios que cursan y por ende, no suelen poseer información que les permita comparar la situación actual, heredada de acciones pasadas, respecto de la nueva situación propuesta en el marco de un proceso de renovación curricular. Más aún, el tránsito entre las distintas asignaturas del plan de estudios, los hace naturalmente más proclives a los cambios, debido a que en cada nuevo periodo académico deben conocer nuevos contenidos y nuevos profesores, con diferentes estilos de enseñanza, que en definitiva se traduce en una actitud más flexible y resignada ante los cambios.

No obstante lo anterior, los cambios siempre generan inquietud. En ocasiones, al menos en la experiencia de la USACH (LCC,2012), ya sea por coyunturas políticas o conflictos en desarrollo, algunos estudiantes interpretan los cambios en el currículo como una amenaza a su carrera. En este contexto, el simple hecho de percibir o sentirse marginados del proceso, puede redundar en una sensación de desagrado y generar un clima de desconfianza que termine impactando negativamente al proceso de renovación curricular. Por tal motivo, es importante incorporar a los estudiantes al proceso de socialización y hacerlos sentirse participes del proceso.

La socialización del proceso en los estudiantes, debe al menos considerar que conozcan: (1) Los objetivos del proceso de modernización curricular del programa y los beneficios que este reportará para los futuros estudiantes, (2) Las diferencias entre la formación profesional o científica con el actual plan de estudios y como el nuevo plan mejora o readecua el proceso del plan vigente, (3) Los efectos positivos tiene la innovación y como beneficia la imagen de la carrera y por ende la de sus egresados.

\section{Socialización en el ámbito Docente}

La incorporación y participación de los docentes en un proceso de renovación o ajuste curricular de un programa, es en la mayoría de los casos el objetivo principal del proceso de perfeccionamiento. Ninguna innovación será exitosa si no contempla acciones y elementos que permitan que cada docente quede en condiciones de cumplir su rol en el contexto del nuevo currículo de la carrera y además, asuma esta tarea como propia. Para la mayoría de los docentes, los procesos de cambio suelen ser fuente de inquietud. Los cambios muchas veces requieren ajustes no solamente en los programas de estudio, sino que en la forma en que los docentes ejercen la docencia. En particular, la educación basada en competencias exige del profesor hacer una reflexión profunda respecto de su ejercicio docente y como debe reorientarse a fin de asegurar que los estudiantes alcancen los estándares definidos para su asignatura.

Por tal motivo, en el ámbito docente, el proceso de socialización debe lograr: (1) Que los docentes reflexionen sobre su práctica docente, los énfasis de los contenidos, el uso de los recursos y las formas de evaluación, (2) Un correcto entendimiento del concepto de perfil de egreso, un conocimiento y entendimiento del perfil de egreso de la carrera y cómo su asignatura aporta a la generación de dicho perfil, (3) Comprensión de los fundamentos del enfoque orientado a competencias, del concepto de competencia, del desempeño, del estándar de desempeño, de los indicadores de desempeño y las condiciones de realización o rúbricas, y (4) Claridad, que como todo proceso de cambio, la introducción o perfeccionamiento en la aplicación del enfoque orientado a competencias, involucrará, al menos inicialmente, más trabajo.

\section{Aplicación}

La aplicación del proceso de perfeccionamiento se realiza conforme al plan de trabajo definido en la etapa de diagnóstico. Debe considerar a todos los actores relevantes e incluir tipos de acciones de 
perfeccionamiento, acordes con los roles de los diferentes actores. Por ende, el tipo de perfeccionamiento, su profundidad o especialización, así como la prolongación en el tiempo, pueden ser muy variados.

No siempre es posible perfeccionar o capacitar a todos los actores del proceso. El plan de trabajo debe dar buena cuenta de estas restricciones y concentrase en aquellos grupos que son de la mayor relevancia para el proceso, tales como, los profesores, los coordinadores de actividades académicas y quienes deban liderar el proceso de innovación.

\section{Perfeccionamiento orientado al Profesor}

El perfeccionamiento orientado al profesor tiene por finalidad lograr que el docente pueda cumplir adecuadamente su rol en el nuevo contexto. Por tal motivo, al finalizar el proceso, el profesor deberá estar capacitado para: 1) Describir los fundamentos del enfoque orientado a competencia, así como el enfoque orientado a competencias influye en las actividades de docencia; 2) Interpretar adecuadamente un programa de estudio diseñado en un enfoque orientado a competencias; 3) Conforme a los estándares de desempeño y las competencias definidas en un programa de la asignatura diseñado en el marco del enfoque orientado a competencias: (a) Planificar las clases, (b) Seleccionar recursos educativos, (c) Definir trabajos y actividades, y (d) construir diversos tipos de actividades evaluativas.

\section{Perfeccionamiento orientado al Coordinador de Actividades Académicas}

En este documento se denomina Coordinador de Actividades Académicas, a la o las personas que realizan la planificación, así como la gestión de las actividades docentes y académicas, de un programa de pre o postgrado. Además, debe monitorear que el ejercicio de la docencia se realice conforme a las orientaciones de la carrera, el perfil de egreso y los programas de asignatura.

Las competencias que el coordinador de actividades docentes debe desarrollar, incluye las dos primeras enunciadas para el perfeccionamiento orientado al profesor y además, sebe ser capaz de: Explicar cómo el enfoque orientado a competencias aporta a la construcción del perfil de egreso de un estudiante; Identificar desviaciones que se produzcan en el ejercicio de la docencia, respecto de las competencias de un programa de asignatura; Aplicar en el proceso de formación profesional o científico, procedimientos básicos de aseguramiento de la calidad de la actividad académica.

\section{Perfeccionamiento orientado a los Diseñadores del Proceso de Formación}

En este documento se denomina diseñadores del proceso de formación, al grupo de especialistas que lideran, analizan, diseñan e implementan un nuevo plan de estudios o una nueva versión del mismo, en el contexto del enfoque orientado a competencias. Este grupo es crucial dentro del proceso de modernización y por tanto, sus integrantes deben tener las competencias necesarias para participar en la construcción del perfil de egreso, definir los itinerarios de formación para los estudiantes (hipótesis evolutiva (Montero, 2008B)), ayudar a los profesores y especialistas a definir los aportes de cada una de las asignaturas al proceso de formación, apoyar la realización de los programas de asignatura en un contexto orientado a competencias, definir posibles mecanismos de aseguramiento de la calidad y monitorear el proceso de implantación de las innovaciones.

Por tal motivo, las competencias que debe desarrollar cada miembro del grupo de diseño del proceso de formación, además de las que debe tener un profesor y un coordinador de actividades académicas, deben ser las siguientes: Identificar fortalezas, oportunidades, debilidades y amenazas de un plan de estudios o carrera; Formular el perfil de ingreso (esperado) en un contexto de enfoque orientado a competencias; Formular el perfil de egreso en un contexto de enfoque orientado a competencias; Conocer como el enfoque orientado a competencias aporta a la construcción del perfil de egreso de un estudiante; Identificar desviaciones en el proceso de formación profesional o científico, que se produzcan en el ejercicio de la docencia, respecto de las competencias de los programas de asignaturas del plan de estudios; Definir e implementar procesos y procedimientos de aseguramiento de la calidad en el proceso de formación.

\section{RECONOCIMIENTO Y CERTIFICACIÓN}

La etapa de reconocimiento y certificación se refiere al proceso mediante el cual la institución, a través de diplomas o certificaciones, así como la realización de eventos o actos formales, preferentemente presididos por autoridades relevantes en el ámbito institucional, reconocen el resultado del perfeccionamiento y certifica la validez y pertinencia del proceso realizado. Diferentes autores (Cáceres, 2006; Mas Torelló, 2011; García, 2014) destacan la importancia de que el perfeccionamiento se realice por vías institucionales. 
La certificación, si bien es una etapa posterior al perfeccionamiento mismo, reviste la mayor relevancia por cuanto representa un fuerte componente motivacional para los participantes, refuerza el compromiso institucional con el proceso y hace explícita la relevancia que la institución le confiere al perfeccionamiento y particularmente al proceso de modernización del programa de estudio [Palominos, 2008]. Los principales efectos positivos del reconocimiento institucional son: La validación el plan de perfeccionamiento, el reconocimiento del esfuerzo de los participantes y la contribución a la carrera académica de los participantes

Los líderes del proceso de modernización curricular deben tener especial cuidado en que en esta etapa se cumpla con todo el rigor y la prestancia que amerita. Se recomienda que el reconocimiento y la certificación del perfeccionamiento, conforme al contexto de cada institución, contemple: (1) La certificación interna y externa, por medio de una resolución o decreto que le confiera al perfeccionamiento valor en la carrera académica, (2) La participación sistemática y explicita de autoridades, en etapas claves del proceso, de modo que su presencia realce la importancia del proceso, y (3) La realización de una ceremonia o acto equivalente, donde además de entregar las certificaciones a los participantes, se destaque públicamente la relevancia del proceso y su importancia para la institución.

\section{CONCLUSIONES}

Las evidencias recopiladas en grupos focales, encuestas y otros trabajos desarrollados en el marco de diferentes proyectos realizados previamente, algunos de cuyos resultados se presentan en (Montero, 2008A; Palominos, 2007) así como pruebas de campo del sistema realizadas a la fecha, dejan en evidencia las siguientes conclusiones:

1) El perfeccionamiento docente y la socialización de los procesos son un factor que en la mayoría de los casos será determinante para el éxito de la innovación y la modernización curricular en el enfoque orientado a competencias.

2) El contexto en el cual se produce la innovación y los aspectos culturales de la institución, son factores que deben ser considerados en la planificación de todo el proceso de perfeccionamiento.

3) El perfeccionamiento debe integrar los elementos conceptuales con los elementos disciplinarios relevantes, de modo que el docente perciba los efectos beneficios del cambio y la innovación.

4) El perfeccionamiento se debe realizar en función del rol del actor en el proceso de enseñanza aprendizaje y debe estar focalizado en aquellos elementos que son esenciales para dicho rol.

5) La evaluación de los aprendizajes se debe revisar y en los casos que corresponda, reorientar hacia aquellos aspectos relevantes respecto de las competencias del plan de estudios y de las asignaturas.

6) El proceso de innovación debe ser bien planificado, con tiempos e hitos acordes con el proyecto, destacándose aquellos aspectos vitales para el proceso de innovación o modernización curricular.

7) Las autoridades universitarias no solo deben acompañar el proceso, sino que además la comunidad académica debe percibirlas como parte integrante del proyecto de innovación, lo que se debe traducir en reconocimiento y respaldo a los participantes y al proceso.

8) El sistema presentado responde a las necesidades previamente mencionadas debido a que es capaz de proporcionar un marco metodológico que responde al requerimiento de identificar adecuadamente las necesidades de perfeccionamiento, considera los factores culturales de la institución donde se aplica, de acuerdo al rol de los participantes, en equilibrio con los aspectos disciplinarios y la evaluación.

\section{AGRADECIMIENTOS}

Este artículo ha contado con la financiación del proyecto "Diseño, desarrollo y prueba de un sistema de perfeccionamiento efectivo para la aplicación de innovaciones en el marco de la educación basada en competencias", de la Dirección de Investigaciones Científicas y Tecnológicas (DICYT), de la Universidad de Santiago de Chile, USACH.

\section{REFERENCIAS}

Angulo, F., Redon, S., Competencias y contenidos: Cada uno es su sitio en la formación docente. Revista de Estudios pedagógicos, vol.37, no.2, p.281-299. ISSN 0718-0705. (2011). 
Cáceres M., M. y otros. La formación pedagógica de los profesores universitarios. Una propuesta en el proceso de profesionalización del docente, Revista Iberoamericana de Educación, ISSN: 1681-5653, (2006).

Camperos, M., La evaluación por competencias, mitos, peligros y desafíos, Foro Universitario, Año 12, № 43, Venezuela, (2008).

Cavallo, D. Models of growth: Towards fundamental change in learning environments, BT Technology Journal $\bullet$ Vol 22 No $4 \cdot$ October (2004).

Climént B., J., Sesgos comunes en la educación y la capacitación basadas en estándares de competencia. Revista electrónica de investigación educativa, 12(2), 1-25, (2010).

Corvalán V., O., Las competencias ya no son lo que eran (ni serán lo que son), Revista Electrónica de Desarrollo de Competencias, Vol. 1, No 1, Instituto de Investigación y Desarrollo Educacional (IIDE), Universidad de Talca, Chile (2008).

Del Valle M., R., Formación por competencias: Certezas, resultados y desafíos, Universidad Católica de Temuco, Innovación Curricular en las Universidades del Consejo de Rectores: Reflexiones y procesos en las Universidades del Consejo de Rectores Prácticas Internacionales, Chile, ISBN: 978-956-7581-03-0. Octubre (2012).

Díaz, B., F., Aportaciones de las perspectivas constructivista y reflexiva en la formación docente en el bachillerato. Perfiles Educativos, vol. XXIV, núm. 98, pp. 6-25, Instituto de Investigaciones sobre la Universidad y la Educación, México, (2002).

Edwards, S H., Hodge, D H. Lessons Learned by Comparing On-line Education Strategies Across Disciplines, Proceedings of the International Conference on Education and Information Systems: Technologies and Applications (EISTA'03), International Institute of Informatics and Systemics, pp. 415420, (2003).

García F., M. D., Modelos de formación y perfil del profesorado universitario: competencias y diferentes estilos,https://www.uco.es/servicios/informatica/windows/filemgr/download/mdgarcia/M.Dolores\%20Garcia\% 20Fdez/texto1.htm. Acceso enero de (2014).

González, L. E., La evaluación de competencias: La experiencia de CINDA, Currículo Universitario Basado en Competencias, Memorias del Seminario Internacional Universidad Del Norte, Barranquilla, Colombia, 25 Y 26 de Julio, (2005).

Guzmán, J. C., Aplicaciones curriculares de la Educación Basada en Competencias (EBC). Balance de la experiencia mexicana a quince años de su implantación. Revista Electrónica de Desarrollo de Competencias (REDEC, Universidad de Talca) - Vol. 2. № 8, (2011).

Herrera, C. y otros. Identificación de las necesidades de capacitación docente de los jefes de programa de especialización médica. Rev. Méd. Chile, Santiago, v. 141, n. 9, sept. (2013).

Jiménez, R. Formación por competencias en la enseñanza de la Arquitectura: “¿Un aporte? ¿Una necesidad? ¿O una moda?. Revista Electrónica de Desarrollo de Competencias (REDEC) Universidad de Talca, N.6. Vol. 2. (2010).

Lewis, B. A., MacEntee, V. M. Learning Management Systems Comparison, Proceedings of the 2005 Informing Science and IT Education Joint Conference, Flagstaff, Arizona, USA, June, (2005).

Mas Torelló, O., El profesor universitario: Sus competencias y formación, Revista de currículum y formación del profesorado, ISSN 1138-414X (edición papel), ISSN 1989-639X (edición electrónica), VOL. 15, 3 http: //www.ugr.es/local/recfpro/rev153COL1.pdf, Diciembre (2011).

Montero, P. y otros autores, Ambientes y Recursos de Aprendizaje Multimediales: Avances y proyecciones de una experiencia en la Universidad de Santiago de Chile, Libro editado por el Centro de Experimentación y Desarrollo de Tecnología Educativa (CEDETEC) de la Universidad de Santiago de Chile (2008A). 
Montero P., Méndez, M., Quezada L., Sepúlveda J., López M., Tarride M., Olivares V., Chacón G. Derpich I., Palominos P., (Depto. de Ingeniería Industrial USACH). "Desarrollo curricular por competencias de Ingeniería Industrial en la Universidad de Santiago de Chile: Hitos de Control del desarrollo evolutivo del estudiante de Ingeniería Civil e Ingeniería de Ejecución Industrial." Ponencia XXII Congreso Chileno de Educación en Ingeniería. La Serena, octubre (2008B).

Moyano D., E., Vásquez R., M., Faundez V., F.. Cambios en el modelo educativo universitario: Desafíos culturales, curriculares y de gestión. Universidad Católica de Temuco, Innovación Curricular en las Universidades del Consejo de Rectores: Reflexiones y procesos en las Universidades del Consejo de Rectores Prácticas Internacionales, Chile, ISBN: 978-956-7581-03-0. Octubre (2012).

Muñoz L., Fernando. La necesidad de regular la docencia universitaria en chile: una propuesta de lege ferenda . Rev. chil. derecho [online]. vol.39, n.3, pp. 891-907. ISSN 0718-3437. (2012).

OECD. Learning Our Lesson: Review of Quality Teaching in Higher Education. (2012).

Palominos, F., Impacto de los ambientes de aprendizaje multimediales y los requerimientos tecnológicos para una implantación exitosa en la enseñanza y formación profesional superior universitaria., Ambientes y Recursos de Aprendizajes Multimediales: Avances y Proyecciones de una Experiencia en la Universidad de Santiago de Chile, Ed.1, CEDETEC-USACH, pp53-75, Santiago, Chile, (2008).

Palominos, F., Implementación de Ambientes de Aprendizaje de Apoyo a la Docencia, Seminario de Evaluación de Competencias en la Educación Superior, organizado por MECESUP, Tuning L.A. y la Universidad Católica de Temuco, Pucón, Chile, Marzo, (2007).

Pedraja R., L., Desafíos para el profesorado en la sociedad del conocimiento. Ingeniare. Rev. Chil. Ing. [online]. vol.20, n.1, pp. 136-144.ISSN 0718-3305. (2012).

Pentland, A, Learning communities : Understanding information flow in human networks, BT Technology Journal, Vol 22, No 4, October (2004).

Pey, R; Chauriye, S. Innovación Curricular en las universidades del Consejo de Rectores, 2000 - 2010. Ediciones CRUCH (2011).

LCC, USACH. Proceso de Autoevaluación, Carrera de Licenciatura en Ciencia de la Computación (LCC), Departamento de Matemática y Ciencia de la Computación, USACH, (2012).

Ramírez, L. V., Medina, G., Educación basada en competencias y el proyecto Tuning en Europa y Latinoamérica Su impacto en México, Ide@s CONCYTEG, Año 3, Núm. 39, Septiembre (2008).

Scallon G. L'évaluation des apprentissages dans une approche par compétences. Saint-Laurent (Montréal): Éditions du renouveau pédagogique (2004).

Tardif, J., Desarrollo de un Programa por Competencias: De la intención a la puesta en marcha. (Traducción de Pedagogie collégiale. Vol. 16. No. 3 Mars por Óscar Corvalán) (2003).

Trujillo J. M., Un nuevo currículum: tecnologías de la información en el aula. Educación y Educadores [online], vol.9, n.1, pp. 161-174, (2006).

UNESCO, Hacia las sociedades del Conocimiento. (2005). 